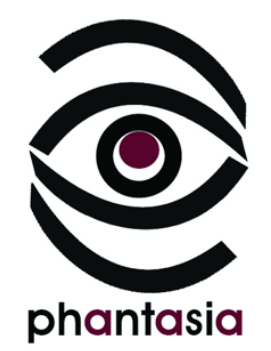

\title{
D'un refuge à un environnement menacé : l'évolution éthique et esthétique de la forêt dans l'œuvre de Pierre Gascar
}

\author{
Sara Buekens \\ UGent
}

Dans Un balcon en forêt de Julien Gracq, l'aspirant Grange découvre le pouvoir incantatoire de la forêt avoisinant le fortin où il est stationné dans les Ardennes françaises. Le désœuvrement de la drôle de guerre lui permet de s'immerger dans cette nature qui sert de décor à ses explorations amoureuses et avec laquelle il finit par éprouver un sentiment de communion. Pour les résistants polonais dans Éducation européenne, un roman de Romain Gary, la forêt est d'abord un refuge qui, malgré les dures circonstances de la vie, symbolise leur espoir pour un monde meilleur, puisque « la liberté est fille des forêts ${ }^{1}$. Pour Pierre Gascar, peu connu à l'époque actuelle et tout à fait oublié des manuels d'histoire littéraire - mais de son vivant aussi renommé et récompensé que Julien Gracq et Romain Gary $^{2}-$, la forêt noue également un rapport très étroit avec l'expérience de guerre. Elle est d'autant plus importante pour l'auteur qu'elle lui a servi de refuge mental et physique lors de sa vie en captivité pendant la Seconde Guerre mondiale.

À partir de ses propres expériences - son séjour dans un fortin en Alsace et sa captivité en Hollande, en Bavière, dans la forêt de Bohème, dans la Ruhr et enfin dans le stalag 325 à Rawa-Ruska en territoire soviétique -, Gascar témoigne dans son œuvre littéraire des capacités thérapeutiques de la forêt, que ce soit lors d'une évasion d'un camp de représailles allemand à travers une forêt-réserve (« La forêt $»^{3}$ ) ou dans un camp de prisonniers où il passe son temps à se promener dans le bois limitrophe ( $L e$ temps des morts ${ }^{4}$, « Les fougères $»^{5}$ ).

Dans cet article, nous étudierons la valeur que l'auteur accorde à la forêt dans les nouvelles et récits où il narre ses propres expériences de guerre. Ensuite, nous nous pencherons sur les descriptions de la forêt dans les romans et récits où il fait preuve d'une grande sensibilité écologique et dénonce la " guerre », aujourd'hui métaphorique, que l'homme et l'industrie ont engagée contre le monde naturel. Dans une perspective écopoéticienne, nous nous concentrerons sur « les questions de forme et d'écriture $»^{6}$ : nous montrerons l'évolution des figures de style, notamment des images et des métaphores, dont

\footnotetext{
${ }^{1}$ Romain Gary, Éducation européenne, Paris, Éditions Gallimard, coll. « Folio », 1972 [1956], p. 57.

${ }^{2}$ Gascar était considéré par Henri Peyre comme un des « six maîtres contemporains » (PEYRE Henri, Six maîtres contemporains, New York, Harcourt, Brace \& World, 1969) et s'est vu décerner un grand nombre de prix littéraires, parmi lesquels le prix Goncourt en 1953 et le prix de l'Académie française en 1969.

${ }^{3}$ GASCAR Pierre, «La forêt » in Le règne végétal, Paris, Gallimard, 1981. Dorénavant FOR.

${ }^{4}$ GASCAR Pierre, Les bêtes suivi de Le temps des morts, Paris, Gallimard, 1953. Dorénavant TM.

${ }^{5}$ GASCAR Pierre, «Les fougères » in Le règne végétal, op. cit. Dorénavant $F G$.

${ }^{6}$ Schoentues Pierre, Ce qui a lieu. Essai d'écopoétique, Marseille, Editions Wildproject, 2015, p. 16.
} 
l'écrivain se sert pour problématiser l'environnement, ainsi que celle des formes stylistiques qu'il juge les plus appropriées à une écriture environnementale. Car on ne saurait oublier que la littérature peut avoir une influence indirecte sur la conscience environnementale du lecteur. Ce n'est pas seulement le contenu du roman mais aussi le travail d'écriture qui détermine sa portée écologique, permettant au lecteur de se réapproprier de nouvelles représentations du monde naturel et de restructurer esthétiquement ainsi que mentalement ses rapports avec l'environnement : " La valeur écologique d'un texte littéraire ne serait donc pas uniquement une question thématique ou une question de choix générique, mais avant tout une question d'écriture, c'est-à-dire d'esthétique et d'imagination, qui sont les critères propres à l'activité artistique $»^{7}$.

Si Gascar est encore connu aujourd'hui, il l'est, dans la plupart des cas, pour ses témoignages autobiographiques sur la mort, la cruauté humaine et la proximité entre l'homme et la bête. Pourtant, les livres de Gascar ne se penchent pas seulement sur la mort : ils questionnent également la vie, et en particulier les secrets du monde naturel. En outre, l'écrivain se montre très sensible à la problématique environnementale. Selon Schoentjes, « [d]ans l'après-guerre, Gascar apparaît comme un des premiers écrivains français reconnus à réfléchir au sort des animaux dans le cadre d'une réflexion sur la protection de l'environnement $»^{8}$. Prêtant également attention aux changements qui frappent le règne végétal à cause de la pollution industrielle, l'auteur manifeste une sensibilité écologique bien avant qu'elle ne fasse partie du discours quotidien. Dans son projet de mise en garde, il défend aussi bien la protection de la flore cultivée que celle du monde sauvage. Ainsi, Gascar fait partie de ces auteurs qui ont exprimé leur attachement au monde naturel et ont mis en exergue dans leurs romans et récits les problèmes environnementaux de leur temps, comme Julien Gracq, Jean-Marie Gustave Le Clézio et Jean-Loup Trassard, à une époque où la critique s'intéressait peu à la littérature ancrée dans le monde concret. Ces auteurs n'ont guère été étudiés dans une perspective environnementale. Maintenant que l'écopoétique apporte les outils stylistiques et narratifs ${ }^{10}$ pour se pencher sur la littérature française dans son rapport avec les questions écologiques, il est temps de revenir sur ces romans et récits qui s'efforcent de saisir le monde dans toute sa matérialité.

\section{D'une réserve paradisiaque à une forêt hantée}

« La forêt », une nouvelle du Règne végétal, relate l'évasion de trois prisonniers d'un camp allemand à travers une forêt qui se révèle être une réserve naturelle. Dès la première phrase, le narrateur autodiégétique évoque une nature dynamique et animée, afin de retracer, dans une esthétique réaliste, l'agitation des fugitifs. Soucieux de répondre aux exigences d'une écriture réaliste, Gascar prête d'autant plus attention aux expériences sensorielles qu'il limite ce que le narrateur observe, et à travers lui le lecteur, au champ visuel caractéristique des promenades en forêt. Traduisant l'expérience concrète des trois compagnons qui s'éloignent le plus vite possible du camp, il décrit le paysage en termes de mouvement, car, selon les lois de l'observation, le mouvement du personnage est perçu par celui-ci comme le mouvement du décor. Au sein de ces observations minutieuses et de ces descriptions détaillées, la forêt se trouve anthropomorphisée par un grand nombre de verbes d'action destinés à transcrire l'expérience de la marche au plus près du vécu. Le lecteur qui court derrière les évadés observe comment l'obscurité « reculait vers le fond de la forêt » (FOR : 129), comment les arbres « se mêle[nt] comme dans une ronde, se superpose[nt]» $(F O R: 131)$, comment la forêt « se renferm[e] sur elle-même, par suite du resserrement des arbres dans la perspective » (ibidem) et est perçue " comme un mur » (ibidem). En mouvement permanent, mais souffrant du double statut d'évadés et d'intrus, les prisonniers

\footnotetext{
${ }^{7}$ Blanc Nathalie, Pughe Thomas, Chartier Denis, « Littérature \& Écologie : vers une écopoétique », Écologie et Politique, n³6, 2008, p. 23.

${ }^{8}$ Schoentues Pierre, Ce qui a lieu. Essai d'écopoétique, op. cit., p. 218.

${ }^{9}$ Par ce terme, nous ne renvoyons pas à ce que l'on désigne aux États-Unis et en ecocriticism par le terme de wilderness, c'està-dire une nature vierge et presque inaccessible à l'homme. Dans les livres de Gascar, il s'agit surtout de végétaux qui existent à l'état sauvage, comme les mauvaises herbes, ou d'animaux sauvages, comme le circaète jean-le-blanc.

${ }^{10}$ Voir à ce sujet : BuEKens Sara, «L'écopoétique : une nouvelle approche de la littérature française », Elfe $X X$ - $X X I, \mathrm{n}^{\circ} 8$, «Extension du domaine de la littérature », sous la dir. d'Alexandre Gefen et Claude Perez, 2019, mis en ligne le 15 septembre 2019, consulté le 20 octobre 2019. Disponible sur : http://journals.openedition.org/elfe/1299.
} 
sillonnent la réserve naturelle avec un grand sentiment d'isolement. Cette influence du mouvement et des sentiments des personnages sur la façon dont le monde naturel est perçu, et, dès lors, décrit, rejoint le point de vue de Marinella Termite qui, dans son analyse de La réticence de Toussaint, montre comment le choix des verbes est susceptible de « guett[er] les références spatiales et [de] les déplo[yer] comme points de repères filtrés. Tout cela favorise un changement de connotations ${ }^{11}$. En outre, Termite affirme que le «sentiment végétal» naît avant tout par une association presque constante des sens ${ }^{12}$. Cette association dépasse largement les connaissances obtenues par l'observation visuelle et subjectivise la perception du monde.

Si les expériences sensorielles se révèlent primordiales pour les fuyards à tout moment attentifs aux signes d'une présence humaine dans la réserve, leur circonspection exerce une influence profonde sur leur expérience du monde naturel. La crainte continue anime non seulement les perceptions visuelles des évadés, mais aiguise aussi leur imagination qui intervient dans un jeu permanent entre le visible et l'invisible :

Elles [les animaux] étaient ici chez elles, tout l'indiquait; d'abord les mangeoires qui, de distance en distance, accrochaient dans l'ombre ces taches claires où, stupéfaits de leur nombre, nous avions été tentés, à un moment, de voir l'effet d'une hallucination, puis le silence, qui trahissait, à n'en pas douter, la présence invisible des cerfs [...], enfin le fait que les gardes-chasse ou les gardes forestiers restaient aussi discrets qu'ils le pouvaient, afin de ne pas déranger les bêtes, en dehors des moments où ils leur apportaient du foin. On pouvait imaginer ces hommes se bornant à nous suivre des yeux à distance, en attendant de nous appréhender au coin d'un fourré, sans avoir besoin de crier et de tirer un coup de fusil en l'air. (FOR : 130-131; nous soulignons)

Nombreux sont les mots qui désignent la perception visuelle, plus nombreux encore sont ceux qui en révèlent l'échec : les animaux et la direction du vent restent " invisibles » (FOR:129; 132 ; $143)$, les réfugiés marchent « en aveugles » (FOR : 135) dans « une masse indistincte » (FOR:131) de troncs au milieu desquels un petit cours d'eau ajoute « à la confusion d'un monde où les feuilles des arbres empêchent de voir la position du soleil » (FOR:136). Or, si les fugitifs se montrent si impatients de discerner les cerfs, c'est d'abord pour voir confirmée par la "liberté » des animaux - une liberté pourtant très restreinte qui de façon ironique se limite aux lisières de la réserve - la réussite de leur propre évasion :

Ce n'était pas la curiosité du promeneur qui nous faisait essayer de les apercevoir, mais plutôt le besoin de nous prouver à nous-mêmes la réalité de notre évasion, de la même façon qu'un voyageur n'arrive à se bien persuader qu'il est en Asie que s'il rencontre un éléphant. La vue de ces bêtes nous aurait aussi rappelé $[\ldots]$ combien étaient naturels, chez tous les êtres vivants, les élans de la liberté. (FOR : 132)

En effet, dans cette forêt domaniale destinée à assurer la survie de certaines espèces animales, les évadés jouissent temporairement de «la même immunité fragile, temporaire, que s['ils s'étaient] trouvés dans un lieu sanctifié, un temple, un cimetière » (FOR : 132). La comparaison avec un espace religieux dote la forêt d'une connotation positive. Ayant échappé à l'inhumanité des camps, les trois fuyards bénéficient maintenant de la protection que paradoxalement les autorités jugent essentielle pour les animaux.

En outre, le contact intime avec l'environnement naturel de la forêt suscite chez les fugitifs un sentiment de bonheur absolu : en tant que lieu de refuge, la forêt devient porteuse de la liberté des hommes en fuite. Le contact physique avec la matérialité des arbres constitue un appui physique autant que mental : si les compagnons dessinent des lignes virtuelles entre les arbres pour retracer un chemin droit « en prenant en somme appui moralement sur un arbre qui venait » (FOR:138), la main touchant l'écorce et sentant la « mince couche velouteuse qui se désagr[ège] sous les doigts » (FOR:146-147) ramène le narrateur à son enfance et lui accorde un moment de paix (FOR : 138). Il n'est donc pas surprenant que l'image du lieu sacré se développe dans une métaphore filée qui met l'accent sur l'intimité du monde clos de la forêt. Ainsi, lorsque les évadés protègent « le nord [de leur boussole

\footnotetext{
${ }^{11}$ Termite Marinella, Le sentiment végétal, Macerata, Quodlibet, 2014, p. 83.

${ }^{12}$ Ibidem, p. 41.
} 
improvisée] comme on protège une flamme » (FOR : 133), leur avancée prend l'image d'une procession « dans un monde où chaque buisson devait lancer de très loin aux habitués des lieux un rappel aussi clair que s'il s'était mis à flamber comme le buisson de la Bible» (FOR : 135). Et si, comme le montre Pierre Schoentjes, le renvoi à la Bible fournit au lecteur des indications sur la culture générale du narrateur ${ }^{13}$ et fait partie des connaissances qui façonnent ses expériences de la nature, les allusions aux histoires surnaturelles des Saintes Écritures ne contribuent pas moins à la création d'un univers magique, où

le tronc [des hêtres] ne portait pas la moindre mousse, comme si [...] ils avaient vécu un autre temps que les saisons de la Bavière et, avec leur écorce grise et lisse, aussi inaltérables que des colonnes de granit, avaient ignoré ce froid et humide climat. [...] je me demandais même s'ils « parlaient » aux habitants de la forêt, s'ils faisaient partie de ceux de ses éléments qui leur étaient familiers, tel ce ruisseau dont nous approchions et entendions déjà le murmure, et dont ils savaient, eux, dans quel sens il coulait. (FOR : 136)

L'anthropomorphisation des éléments naturels dépasse ici largement sa fonction de " faire voir $»^{14}$ et ne tient plus seulement à l'effet du mouvement des personnages. Dans l'imagination du narrateur, les hêtres deviennent les colonnes vivantes d'un temple dans une forêt animée où les végétaux, les animaux et les éléments communiquent comme dans un passé lointain et paradisiaque ${ }^{15}$.

$\mathrm{Au}$ fur et à mesure que les évadés pénètrent au cœur de la réserve, lorsque la crainte d'une présence invisible l'emporte sur la part de réalité perceptible et que l'imagination prend la liberté de combler les vides, la forêt perd de plus en plus son caractère réaliste. Coupée du monde extérieur, la réserve constitue un univers à part sur lequel l'histoire récente semble n'avoir eu aucune prise et s'assimile à un endroit hanté qui bouleverse les lois de la réalité environnante.

Dans les évocations de la forêt, des animaux et des végétaux qu'elle abrite ou des circonstances climatologiques, des indices toujours plus nombreux révèlent un monde irréel dans lequel les règles de la logique n'ont plus leur place. Ainsi, les évadés remarquent que « [1]e ciel s'assombrissait plus vite que l'heure ne le justifiait » (FOR : 145). Les observations minutieuses du début de la nouvelle sont devenues des métaphores qui engendrent un effet de « déréalisation»:

On eût dit que l'air s'y décantait à la façon d'un liquide dans lequel un tournoiement, une agitation, eût maintenu auparavant en suspension mille corps presque imperceptibles qui descendaient maintenant reposer sur le fond, rendant la transparence à l'élément qu'ils avaient troublé et que leur chute ne débarrassait pas seulement de leur présence, mais, comme jouant le rôle d'un filtre, par surcroît, purifiait. (FOR : 139)

Se servant d'un vocabulaire mécanique, presque chimique, le narrateur évoque un univers abstrait où de nouvelles forces cosmiques font interagir tous les éléments naturels.

En outre, errant dans « un piège qui tenait de l'ensorcellement » (FOR:148), les trois compagnons se voient harcelés par des arbres qui, par magie, les condamnent à toujours tourner en rond (FOR : 149) : « je rêvais peut-être... [...] Cependant, maintenant que j'avais dépassé ce "revenant", je n'en finissais pas de tourner la tête en arrière, afin de m'assurer, une fois encore, de sa réalité » (FOR : 150). Les fugitifs ont beau aiguiser leurs sens dans cette forêt « dont la réalité [...] paraissait de plus en

\footnotetext{
${ }^{13}$ Pierre Schoentjes montre qu' « il n'existe jamais d'expérience de la nature au présent. L'expérience actuelle du narrateur dans "La forêt" se fait par le truchement de relais culturels forts qui invitent tout un savoir dont la constitution est bien antérieure à la naissance du narrateur ». SCHOENTJES Pierre, Ce qui a lieu. Essai d'écopoétique, op. cit., p. 206.

14 «Le but que je m'efforce d'atteindre est, avec le seul pouvoir des mots écrits, de vous faire entendre, de vous faire sentir, et avant tout, de vous faire voir. » CONRAD Joseph, Préface à Le nègre du « Narcisse », trad. R. d'Humières, Paris, Gallimard, 1938 [1898], p. 7. Schoentjes cite Conrad lorsqu'il repère chez Gascar une esthétique du sensible qui «nomme les choses avec précision, décrit le monde physique avec une attention pour le détail concret ». SCHOENTJES Pierre, Ce qui a lieu. Essai d'écopoétique, op. cit., p. 204.

${ }^{15}$ Remarquons que cette citation permet aussi de situer l'expérience du narrateur dans la sphère des contes, un rapprochement justifié en outre par la description du garde-chasse qui intercepte les fugitifs et qui semble sortir d'un conte de fées des frères Grimm : «Avec sa veste verte, son chapeau bavarois, ses guêtres et sa barbiche grise, il n'appartenait pas seulement, et de la façon la plus attendue, au monde forestier, mais aussi à un temps très éloigné du nôtre, comme s'il sortait d'une de ces images allemandes du siècle dernier où, assis devant la porte d'un pavillon de chasse fait de rondins, il aurait fumé sa pipe de porcelaine » (FOR : 141-142).
} 
plus incertaine » (FOR : 144), les « châtiments des contes » (FOR:152) transforment l'évasion en un véritable cauchemar. La forêt est un univers réservé aux animaux et aux végétaux, où l'homme n'a pas sa place et où les intrus, en dépit de leur euphorie initiale, se voient menacés par une nature hostile.

Ce n'est qu'au moment où le narrateur, souffrant d'une douleur lancinante au pied, abandonne son projet d'évasion et décide de rester sur place, à la lisière du bois, puis, plus tard, de gagner le village le plus proche pour s'y rendre, que la forêt respire à nouveau « la paix, invit[e] [...] au repos » (FOR : 154). Le narrateur a cessé d'utiliser la forêt pour son propre profit : il veut maintenant découvrir la beauté et les secrets des végétaux et des animaux qui la peuplent. À partir de ce moment, le narrateur ne valorise plus la forêt utilitaire, c'est-à-dire en tant que refuge, mais considère la nature pour elle-même. La forêt se transforme à nouveau en un univers bienveillant qui se situe une fois de plus dans la sphère édénique : un " monde à demi irréel qui tient un peu de celui de l'enfance [...] un paradis intérieur » (FOR : 155).

\section{Une forêt guerrière}

Dans Le temps des morts ainsi que dans « Les fougères », deux récits où Gascar témoigne de son séjour dans un camp de représailles à Rawa-Ruska, la forêt apparaît également comme un lieu de refuge. Chargé de l'aménagement et de l'entretien du cimetière, le narrateur a l'occasion, ne fût-ce que pendant de brèves escapades, de pénétrer dans la forêt pour ramasser du bois. Même s'il ne s'agit pas d'une " évasion » définitive du camp, il découvre la paix qu'exhale la nature, devenue porteuse du bonheur d'hommes libres : " Nous redécouvrions soudain les images de notre liberté éphémère, cette liberté du fugitif, où la réalité, ici celle de la nature, comme avivée par la joie, l'impatience et l'angoisse, se peint d'irremplaçables couleurs » $(F G: 17)$. Suscitant des pensées nostalgiques, la nature ne reflète pas seulement le bonheur temporaire des prisonniers, elle exerce également une influence sur l'esprit des hommes : «Quels souvenirs de liberté, quelles images heureuses, cette paix et cette lumière du sousbois ramenaient-elles dans notre esprit ?» $(F G: 18)$. Il s'agit donc d'une juste réciprocité : si les prisonniers projettent leur exaltation sur la forêt, celle-ci à son tour provoque chez eux sa part d'émotions.

Le pouvoir enchanteur de la forêt ne se limite d'ailleurs pas aux prisonniers privés de toute forme de liberté : le gardien qui les accompagne jouit autant de la paix qu'exhalent les grands arbres. Oubliant les mornes couleurs du camp et le regard triste des habitants de la ville de Rawa-Ruska, il « avançait $[\ldots]$ comme perdu, lui aussi, dans un rêve » $(F G: 18)$. Si les prisonniers arrivent à mener dans cette forêt, sous l'œil bienveillant du gardien, une vie de boy-scout, cueillant des baies, mijotant des champignons dans une gamelle et, assis autour d'un arbre, identifiant les oiseaux à leur chant - des activités qui ne sont pas sans rappeler la vie paysanne des soldats d'Un balcon en forêt -, c'est parce que cette forêt constitue pour tous les êtres humains ce lieu édénique et universel où la beauté de la nature l'emporte sur les atrocités commises par l'homme :

On se serait cru dans n'importe quel bois de la Bourgogne ou du Wurtemberg, quelques années plus tôt, avant la guerre, dans un monde dont nous pensions, depuis que nous étions soldats, qu'il avait à jamais disparu. [...] nous redécouvrions maintenant sous ces arbres, intact depuis nos dernières vacances, un peu du paradis perdu de notre adolescence, encore si proche dans le temps. À défaut de nous offrir une réelle possibilité d'évasion, [...] la forêt représentait pour nous, et aussi semblait-il, pour ce soldat allemand chargé de notre surveillance, le lieu d'une secrète désertion. (FG:18-19)

Or, même si les souvenirs que font naître la vue, l'odeur et le frôlement des arbres créent généralement un sentiment de protection et de réconfort, et replongent les prisonniers dans un monde rassurant, la nature n'est pas toujours à même de chasser les souvenirs des pires horreurs de la guerre. Lorsque les images d'actes inhumains reviennent à l'esprit des captifs, l'environnement naturel offre une image aussi attristante que les trains partant pour les camps d'extermination. Comme dans les œuvres de Gracq, où la nature devient une caisse de résonance pour les événements décrits ${ }^{16}$, la nature

\footnotetext{
${ }^{16}$ Leutrat Jean-Louis, Gracq, Paris, Éditions Universitaires, coll. « Classiques du XX ${ }^{\mathrm{e}}$ siècle », 1967, p. 40.
} 
du monde concentrationnaire décrite par Gascar incarne les signes de la guerre : « Alentour, le vide et le silence de la campagne blafarde disaient autant la guerre que le grondement lointain d'une canonnade l'eût fait. La peur avait arrêté la vie » $(F G: 13)$. La guerre a beau être matériellement absente, les endroits abandonnés par la population cessent de parler de paix et d'intimité. Tout comme dans « La forêt ", Gascar se rend compte que, si au début la nature a offert à l'homme une image réconfortante, celle-ci n'est que temporaire et trompeuse : « Nous avions effectivement tort de croire aux images du jour, de croire à ce que suggérait la petite vallée [...]. Il fallait que la guerre habitât d'avance le paysage, nous habitât entièrement, comme déjà elle habitait nos nuits » (FOR : 27). Ainsi, la guerre n'épargne pas le monde naturel et finit par tout uniformiser « jusqu'aux éléments, l'eau, la terre, le nord, le sud, le jour, la nuit » (FOR : 70).

La forêt s'assimile d'autant plus aux menaces de la guerre qu'elle se révèle l'endroit par excellence où l'homme projette ses émotions sur la nature environnante. Même les souvenirs d'enfance les plus heureux n'arrivent pas à chasser la hantise de l'univers concentrationnaire : "Cette forêt où le printemps allumait, un instant plus tôt, la mémoire, voici que de la peur et de la faim des hommes elle jaillissait plus sombre, plus serrée, plus mystérieuse et plus propice comme un plissement hercynien. La peur foudroie la réalité » $(T M: 223)$.

Comme l'a remarqué Robbe-Grillet dans La Nouvelle Revue française, le lecteur du Temps des morts est " assourd[i] par le symbole $»^{17}$. Les réalités naturelles, les paysages et en particulier les phénomènes météorologiques jouent le rôle annonciateur d'un péril imminent. Ainsi, l'image de la mort est présente dans « le poids de l'heure, la couleur du ciel, les lignes du paysage » (TM:213), les menaces se lisent dans le ciel (TM:240), où l'absence d'oiseaux ranime la mythologie des mauvais auspices $(T M: 251)$ et où l'opposition symbolique de la lumière et des ténèbres se concrétise dans « le flux de la lumière [...] impuissant à dissiper [...] les images terrifiantes de l'ombre » $(T M: 240)$. Les descriptions du monde naturel superposent de la sorte des images ancrées dans la matérialité du monde sensible et des présages apocalyptiques qui tendent vers un réalisme onirique :

Les herbes, disait Cordonat, ce sont les cheveux blancs des morts, paroles qui témoignaient du culte sénile dans lequel nous nous enfoncions de plus en plus, à mesure qu'autour de nous, augmentaient les menaces. [...] Mais c'était surtout le silence grandissant de l'été, la pâleur du soleil, à certaines heures, la chaleur oppressante qui nous alarmaient secrètement. (TM:256)

La citation montre comment, dans ces descriptions d'une forêt envahie par la guerre, les éléments et les végétaux sont souvent présentés par une association surprenante de métaphores et, comme les hêtres de «La forêt», dotés de qualités humaines. Cette anthropomorphisation ne leur permet pas seulement d'interagir entre eux, mais aussi de réagir, à leur tour, aux événements historiques. Ainsi, lors d'un orage, les tournesols incarnent le désir de contestation des prisonniers, prenant « une telle luminosité [...] comme se répondant, qu'on avait l'impression d'assister à la brève revanche tapageuse d'un peuple végétal et condamné le reste du temps à la grisaille du soleil » $(T M: 213)$, alors que le buisson « se plaint à voix humaine » $(T M: 218)$. Cette liberté poétique s'explique en partie par le rôle que la nature, dans Le temps des morts ainsi que dans " Les fougères ", joue pour Gascar lors de son emprisonnement : d'une part, elle constitue un refuge où l'auteur peut imaginer un univers coupé de la réalité de la Seconde Guerre mondiale; d'autre part, le monde naturel sert de caisse de résonance à ses sentiments d'horreur, qu'il ne peut traduire que par le biais d'un langage poétique :

Les mirages de la nostalgie sont capables de supplanter bien des paysages. Celui au milieu duquel nous nous trouvions commençait, toutefois, de se dessiner avec tant de cruauté qu'il pouvait transpercer toutes les fausses images : c'était sur une herse que mes compagnons couchaient leurs rêves. (TM: 222-223)

Ainsi, l'écriture de Gascar participe à ce phénomène de «projection » que Michael Bess décrit dans La France vert clair : « [c] ]haque époque tend à projeter ses métaphores, ses peurs et ses désirs sur

\footnotetext{
${ }^{17}$ RoBbe-GriLlet Alain, «Pierre Gascar : Le temps des morts (Gallimard) », La Nouvelle Revue française, $\mathrm{n}^{\circ} 13$, janvier 1954, p. 138.
} 
la nature qui l'environne ${ }^{18}$. Comme le montre Andrée Corvol dans L'homme aux bois, les forêts ont toujours été importantes pour les Français aux niveaux économique, sociologique et militaire, et elles constituent de la sorte un "miroir de la société ${ }^{19}$. Enracinée dans l'imaginaire de la culture française, la forêt se prête facilement à des projections de joie, de peur, de désolation ou de colère, surtout lors d'une situation extrême comme celle de la guerre.

\section{Une forêt menacée}

La forêt joue encore un rôle important dans les récits ultérieurs de Gascar, dans Les sources ${ }^{20}$ et Pour le dire avec des fleurs ${ }^{21}$, au sein desquels elle apparaît comme une entité menacée dans son existence. Devenue elle-même une victime de guerre, c'est-à-dire un objet détruit par la société qui s'industrialise, elle est désormais décrite à l'aide du vocabulaire qui s'appliquait avant aux prisonniers des récits sur la Seconde Guerre mondiale : l'homme se rend « maître » de la nature $(L S: 60)$ qui fait preuve de « divers degrés de résistance » $(P L D: 40)$ et les quelques survivants végétaux ne sont que des " rescapés ou, plus exactement, [d]es sursitaires » (ibidem). Si l'homme continue son " œuvre d'extermination $»^{22}$, les arbres, dont les branches sont trop souvent «amputées » (PLD:117) et qui ont de plus en plus de mal à « survivre » $(P L D: 61)$, finiront eux aussi par tomber dans des " génocides végétaux » $(P L D: 53)$.

En effet, comme le montre Gascar en 1975 dans Les sources, l'inflation économique de l'époque, engendrant une augmentation des prix des combustibles, a causé la disparition progressive de forêts entières réduites en bois de chauffage. Or, les coupes de bois qui s'effectuent à l'intérieur des forêts - dans la plupart des cas, l'abattage ne s'effectue pas à la lisière $(L S: 63)$ - ne laissent pas apparaître tout de suite les dommages causés au paysage français. De ce fait, l'abattage s'est d'abord déroulé à l'insu de la population, "favorisant l'érosion, modifiant les climats, réduisant nos réserves forestières, et ne s'accompagn[ant] que d'un reboisement partiel et d'une nature des plus discutables » $(L S: 65)$. De grandes landes s'étendaient désormais à l'intérieur du « bois du Molain, bois de la PierreEncize, bois des Prés Bergeret, bois de la Combe noire " $(L S: 63)$, où le paysage sylvestre a pris une couleur champêtre. Ce n'est qu'au moment où le gouvernement, régi par le profit financier immédiat, a décidé de substituer des sapins aux arbres abattus, que le caractère d'un grand nombre de régions en France s'est trouvé profondément modifié.

Outre les modifications esthétiques et les transformations du mode de vie des paysans contraints de changer leurs cultures à cause de la proximité des forêts de sapins, la plantation de résineux, " couverture préboréale » $(L S: 72)$, provoque aussi des altérations profondes dans la nature. Le sol sur lequel les plantations s'accumulent devient vite stérile et trop acide pour la plupart des plantes sauvages. Recouvert d'aiguilles, il n'abrite que peu d'animaux (PLD : 113). De plus, les modifications météorologiques dues à l'enrésinement des régions françaises sont nombreuses : « brouillards fréquents, détournement des vents, surcroît d'humidité et les transformations concomitantes de la lumière du jour » (PLD : 116).

Or, dans ce discours écologique, la nature n'est pas la seule victime : l'homme industrialisé aussi est prisonnier de la société de consommation et la forêt lui offre encore autant de soulagement que lors de la Seconde Guerre mondiale. Aujourd'hui devenu un impératif, le progrès modifie le monde à une vitesse fulgurante, de sorte que l'homme ne s'y retrouve plus. Gascar donne l'exemple des lourdes machines agricoles qui, imposant "une image de conquête » $(L S: 21)$, envahissent des villages traditionnels comme Baume-les-Messieurs. Le monde familier est en train de disparaître et l'individu se perd. Selon Gascar, l'homme industrialisé tente alors de reprendre pied en rétablissant ses anciens rapports avec la nature : il s'agit d'un « tellurisme qui restera un des traits marquants de notre civilisation

\footnotetext{
${ }^{18}$ Bess Michael, La France vert clair. Ecologie et modernité technologique 1960-2000, trad. Chr. Jaquet, Paris, Champ Vallon, 2011 [2003], p. 79.

${ }^{19}$ Corvol André, L'homme aux bois. Histoire des relations de l'homme et de la forêt XVII $-X X^{e}$ siècle, Paris, Fayard, 1987, p. iv

${ }^{20}$ GASCAR Pierre, Les sources, Paris, Gallimard, 1975. Dorénavant $L S$.

${ }^{21}$ GaSCAR Pierre, Pour le dire avec des fleurs, Paris, Gallimard, 1988. Dorénavant $P L D$

${ }^{22}$ Gascar Pierre, L'homme et l'animal, Paris, Albin Michel, 1974, p. 11.
} 
déclinante » (LS:85). Dans La friche, par exemple, Gascar décrit l'exode régulier des citadins vers la campagne, un exode qui n'épargne pas son village. Ces citadins achètent des résidences pour échapper à l'agitation urbaine et pour redécouvrir la simplicité d'une vie en harmonie avec la nature environnante. Ainsi, « [1]es nouveaux venus au village, tout destinés qu'ils sont à être remplacés un jour, par d'autres, car l'exode vers la nature et le loisir ne peuvent que s'amplifier, n'annoncent jamais qu'un avenir statique. Mais n'est-ce pas là déjà un aboutissement ? ${ }^{23}$. Automatiquement, ce retour à la nature, sans pour autant incarner un renouveau du romantisme ou une initiation au mode de vie des sociétés primitives, symbolise l'espoir que garde l'homme de retrouver « à l'intérieur de sa triste condition humaine un rêve consolateur $»^{24}$. Et ce havre tranquille, l'homme moderne le trouve toujours dans la forêt qui lui permet de renouer avec ses anciens rapports au monde naturel et qui n'a jamais cessé de constituer un endroit de paix et d'espoir :

On accompagne, par la pensée, les premières pousses qui apparaissent, un beau jour, dans une saignée de la falaise où bientôt une coulée de verdure s'étendra, pour donner, au bout de quelques années, des arbres noueux, croissant presque à l'horizontale dans le vide. Il n'est personne que cette invasion végétale ne satisfasse, en secret, comme si, fatiguée des déserts que ses progrès et sa science lui ouvrent, l'humanité aspirait confusément à retrouver l'ombre, à regagner le couvert. $(L S: 51)$

\section{Une forêt scientifique}

Jusqu'ici, nous nous sommes demandé dans quelle mesure la forêt pouvait avoir un effet régénérateur pour l'être humain en détresse. Selon Gascar, il faut également inverser la question : la nature est-elle sensible à ce qui se passe autour d'elle ? L'homme a-t-il une influence sur les sensations des végétaux ? Faut-il s'émouvoir des coups de cognée des bûcherons et avoir pitié des souches des taillis ? Grand lecteur de Césalpin, Gascar décrit comment, déjà au $16^{\mathrm{e}}$ siècle, ce botaniste a découvert dans les végétaux un centre vital ou nerveux qu'il appelait " âme » (PLD:24). Aujourd'hui, montre Gascar, en mesurant les radiations et l'énergie émises par les plantes, les scientifiques ont constaté qu'il existe une sorte de télépathie entre les végétaux et donc, conclut-il, « une forme embryonnaire de la conscience » $(P L D: 33)$. La description de cette conscience permettra à l'homme de découvrir « comment les rapports de la matière animée avec le milieu se complexifient, selon les degrés d'évolution, comment la nature passe d'une vie purement biologique à une vie déjà personnalisée » $(L S$ : 70). Or, ce n'est ni par aspiration anthropomorphiste ni par sentimentalisme que les sciences cherchent à découvrir dans les végétaux une " activité nerveuse » $(L S: 70)$ ou même une " mémoire » $(L S: 70)$, c'est-à-dire une "faculté de prévision » $(L S: 70)$ qui incite les scientifiques à parler de "phytopsychologie » $(L S: 70)$. Par conséquent, l'existence d'une conscience végétale oblige l'homme à étendre la communauté biotique et à inclure les arbres dans la catégorie des " autres » qui font appel à sa sensibilité éthique et écologique et envers lesquels il doit être capable de justifier ses actions. Selon Gascar, « [n]ous rentrons dans un monde unanime où l'insecte cogite, où l'herbe soupire, où l'arbre écoute, où le rocher lui-même recèle un esprit ; un monde où, à chacune de nos découvertes, apparaît un peu plus d'unité, un peu plus de fraternité » (LS: 68-69). Dès lors, tous les éléments de l'écosystème sont mis sur un pied d'égalité et l'environnement non humain est plus qu'un simple décor : tout organisme (végétal, animal ou humain) est un protagoniste avec ses propres intérêts ${ }^{25}$.

\footnotetext{
${ }^{23}$ GASCAR Pierre, La friche, Paris, Gallimard, 1993, p. 82.

${ }^{24}$ GASCAR Pierre, «Une civilisation qui retourne à l'école des fleurs », in Pierre Gascar et Guy Rohou. Correspondance. 19631995, Bibliothèque d'Etude et de Conservation de Besançon, fonds Pierre Gascar, Ms. Z 481.

${ }^{25}$ Ainsi, l'oeuvre de Gascar répond aux critères établis par Lawrence Buell pour définir le texte « environnemental » :

1. L'environnement non humain est présent non seulement comme cadre, mais comme une présence qui suggère que l'histoire humaine s'inscrit dans l'histoire de la nature ;

2. L'intérêt humain n'est pas considéré comme le seul intérêt légitime ;

3. La responsabilité de l'homme envers l'environnement fait partie de l'orientation éthique du texte ;

4. Le texte appuie au moins implicitement une conception de l'environnement comme processus plutôt que comme constante. (BuEll Lawrence, The Environmental Imagination, Cambridge/London, Harvard University Press, 1995, p. 7-8, nous traduisons.)
} 
Remarquons que cette idée de communication végétale, qui fait écho aux conversations des hêtres évoquées dans «La forêt » et aux plaintes du buisson dans Le temps des morts, n'a plus rien à voir avec l'évocation anthropomorphique d'un univers hanté qui traduisait la peur des prisonniers. Comme le montre Romain Bertrand, l'emploi de termes référant à des comportements humains a permis à de nombreux scientifiques non pas de décrire le monde naturel dans une perspective anthropocentrique, mais de montrer que les règnes végétal et animal sont particulièrement complexes et intelligents et que « certaines propriétés habituellement associées à l'homme [...] ne lui appartiennent en rien $»^{26}$. C'est donc dans une perspective antispéciste ${ }^{27}$, qui consiste à attribuer une valeur égale à tous les organismes vivants, et sur base de découvertes scientifiques, que Gascar révèle une sensitivité végétale. Ainsi, dans les derniers récits de Gascar, les associations poétiques ont cédé la place à un discours qui s'inspire ouvertement de données provenant des sciences naturelles. Cette évolution des modes de représentation de la forêt et des réflexions qu'elle suscite au fil de la production littéraire de Gascar s'inscrit dans la transformation stylistique générale de son œuvre ${ }^{28}$. L'auteur, qui s'intéresse de plus en plus à l'histoire naturelle, échange le monde concentrationnaire de ses premiers romans et récits contre des descriptions détaillées et scientifiquement étayées de la beauté et de la valeur de la nature. Cette évolution, déjà visible dans Le règne végétal (le recueil contenant " La forêt " et "Les fougères $\left.»^{29}\right)$, s'accompagne d'un abandon progressif des figures de style qui produisent un effet " déréalisant ». L'anthropomorphisme, le symbolique et l'onirique cèdent la place au discours produit par la biologie, la géographie, l'histoire naturelle, la botanique...Comme le juge Gascar, ce dépouillement stylistique est indispensable pour décrire, de façon véridique et appropriée, la problématique environnementale et pour rendre compte des altérations qu'ont causées le progrès et l'industrialisation. Selon Schoentjes ${ }^{30}$, Gascar est revenu pour une raison similaire sur son témoignage au sujet des camps de représailles consigné dans Le temps des morts, qu'il a réécrit en 1998 dans $L e$ rêve russe. Dénué de tout embellissement lyrique et poétique, celui-ci répond mieux aux attentes - une approche sérieuse et respectueuse - d'un témoignage de guerre qui ramène l'attention sur l'éthique plutôt que sur l'esthétique : "Avec le recul il paraît inacceptable à Gascar d'avoir "fait de la littérature", en d'autres termes, d'avoir produit un objet esthétiquement travaillé dont le mérite revient à l'artiste, en exploitant la souffrance qui a été celle des Juifs, gazés ou exécutés par la balle ${ }^{31}$. « Les fougères », qui relate le même épisode, constitue déjà en 1981 une version corrigée dans ce sens.

En insérant des données scientifiques dans ses œuvres littéraires, Gascar pratique un procédé toujours actuel et de plus en plus répandu : on observe dans les romans et récits " environnementaux » de l'extrême contemporain de nombreuses références aux sciences naturelles, qui permettent de décrire les problèmes écologiques dans un discours largement reconnu comme incontestable et « objectif », même à l'intérieur d'une œuvre d'art (pensons au Règne du vivant d'Alice Ferney, à L'aménagement du territoire d'Aurélien Bellanger ou à Autour du monde de Laurent Mauvignier). Si déjà au $19^{\mathrm{e}}$ siècle,

\footnotetext{
${ }^{26}$ Bertrand Romain, Le détail du monde. L'art perdu de la description de la nature, Paris, Éditions du Seuil, 2019, p. 150.

${ }^{27}$ Le spécisme (« speciesism ») est un terme introduit par Richard Ryder en 1970 pour désigner toute forme de discrimination et instrumentalisation d'une espèce (animale) justifiée par l'idée que l'homme est un être supérieur. Développée par Peter Singer, la notion est entre-temps utilisée surtout dans sa forme négative (« anti-speciesism ») dans les nombreux discours défendant les droits des animaux et promouvant le végétarisme (BEKOFF Mark, Encyclopedia of animal rights and animal welfare. Second edition, Volume I : A-H, Santa Barbara (California), Greenwood Press, 2010, p. 527-528).

${ }^{28}$ Voir à ce sujet : Buekens Sara, «Pour que l'écologie supplante le nationalisme : l'esthétique de Pierre Gascar », Revue critique de fixxion française contemporaine, $\mathrm{n}^{\circ} 11$, « Écopoétiques », sous la dir. d'Alain Romestaing, Pierre Schoentjes et Anne Simon, décembre 2015, p. 49-59.

${ }^{29}$ Dans « Les fougères », on sent bien qu'outre ses expériences de guerre, Gascar veut également transmettre sa fascination pour le monde naturel, dont il montre la richesse à l'aide d'énumérations et de données scientifiques : « Tardant à m'endormir, car quelques puces bravaient toujours l'odeur et la fraîcheur de la fougère, je m'amusais dans l'obscurité à distinguer au toucher les feuilles fertiles de celles qui ne l'étaient pas. Les premières - je l'avais appris en classe de sciences naturelles - portent sur leur envers des sporanges qui leur donnent le relief, le grain d'une broderie, d'un galon. Chez les autres plantes, les graines sont logées au cœur des fruits, dans des capsules, des gousses, des épis, ou se présentent en grappes, en ombrelles, en corymbes, etc. Ici, elles envahissent l'ensemble de la fronde, comme les myriades de pucerons qui couvrent quelquefois toutes les parties d'un rosier : un grouillement figé. L'idée de grouillement ne me serait pas venue à l'esprit, si je n'avais su que, dans la reproduction des fougères, apparaît une gamète pourvue de flagelles dont, à la faveur de la pluie, elle se sert pour nager vers l'organe féminin présent dans le petit organisme de la spore » $(F G: 30)$.

${ }^{30}$ SchoentJes Pierre, «Pierre Gascar : retour sur Le Temps des morts», in Mémoires occupées. Fictions françaises et Seconde Guerre mondiale, sous la dir. de Marc Dambre, Paris, Presses Sorbonne Nouvelle, 2013, p. 101-110.

${ }^{31}$ Ibidem, p. 103.
} 
pour des auteurs comme Balzac, le savoir scientifique a servi à légitimer la littérature " comme appropriation et/ou explication du monde $»^{32}$, de nombreux romans et récits de la seconde moitié du $20^{\mathrm{e}}$ siècle et du $21^{\mathrm{e}}$ siècle n'hésitent pas à se définir explicitement comme lieux de savoir, brouillant constamment les frontières entre « réel et fiction, littéral et figural, suite de résultats scientifiques et imaginaire $»^{33}$. Aussi cet enrichissement mutuel entre discours scientifiques et pratiques littéraires, par lequel les œuvres de fiction centrées sur la problématique environnementale cherchent à gagner en sérieux, a-t-il fortement influencé les humanités environnementales qui se présentent comme un «pont interdisciplinaire entre la science et la critique littéraire ou culturelle $»^{34}$. En effet, comme le montre Ursula Heise, les sciences naturelles ont contribué de manière significative à l'évolution de la pensée écologiste depuis les années 1960 et ont permis de renforcer la crédibilité du mouvement environnementaliste auprès du grand public.

Christian Chelebourg analyse dans Les écofictions la façon dont les différents discours actuels (parmi lesquels les œuvres de fiction) intègrent les thèses et les questions environnementales dans leurs narrations. Au début de son livre, il résume le « fameux schéma narratif ${ }^{35}$ qui structure les œuvres romanesques intégrant des préoccupations écologiques :

situation initiale, l'harmonie de la nature ; élément perturbateur, l'industrialisation humaine ; péripéties, les dégâts environnementaux ; élément de résolution, la recherche en écologie ; situation finale, désastre ou salut, au choix, selon que l'on aura bien ou mal agi, selon que l'on aura pris ou non les bonnes décisions, adopté ou non les bons comportements ${ }^{36}$.

Pourtant, ce schéma ne s'applique pas aux derniers récits de Gascar, dans lesquels le narrateur continue toujours à vivre en harmonie avec la nature, les dégâts environnementaux ne sont pas irréversibles et la solution reste incertaine. Gascar ne se montre ni pessimiste ni catastrophiste : conscient des bienfaits de la modernité et convaincu qu'il est possible encore pour l'homme d'adopter un autre mode de vie, plus respectueux du monde naturel, il ne cherche nullement à prôner l'ancienne opposition romantique entre culture et nature. La structure décrite par Chelebourg ne constitue donc qu'une seule possibilité parmi les milliers de cas étudiés par l'écopoétique ${ }^{37}$. Ainsi, John Fowles aborde dans The Tree la problématique du déracinement de l'homme moderne qui, dans sa rage de vivre, oublie la plusvalue qu'apporte le monde naturel à son existence. Contrairement à Gascar, Fowles propose un rapprochement avec la nature qui consiste à écarter toute forme de savoir scientifique. Selon cet auteur anglais contemporain de Gascar, les sciences naturelles incitent l'homme à s'éloigner de la nature, traitant les règnes végétal et animal « comme une sorte de rébus intellectuel, de puzzle ${ }^{38}$. L'étude scientifique du monde naturel, qui consiste en l'analyse de ses différents composantes et l'explication de ses mécanismes, "pla[ce] insidieusement la nature dans une situation d'une sorte d'opposant, d'équipe adverse qu'il conv[ien]t de vaincre en se montrant plus malin qu'elle $»^{39}$. Ces questions réductrices, inconciliables avec la complexité de l'expérience de la nature, interdisent à l'homme d'entrer spontanément dans l'intimité de ce qui l'entoure ainsi que d'éprouver une « expérience totale » ${ }^{40}$ de la nature. Cette position a évidemment des répercussions au niveau de l'écriture : là où les textes de Gascar sont truffés d'informations provenant de l'histoire naturelle, Fowles écarte toute donnée scientifique pour défendre sa cause.

\footnotetext{
${ }^{32}$ ACQUIER Marie-Laure, «Présentation : soif de mots, désir de science. Quelques aspects de la relation entre littérature et science(s) », Cahiers de Narratologie, 18, 2010, mis en ligne le 16 juillet 2010, consulté le 20 octobre 2019. Disponible sur : $\mathrm{http}: / /$ narratologie.revues.org/6116.

${ }^{33}$ Ibidem.

${ }^{34}$ Heise Ursula, « Science and Ecocriticism », American Book Review, 18, n5, 1997, p. 4.

${ }^{35}$ Chelebourg Christian, Les écofictions. Mythologies de la fin du monde, Bruxelles, Les Impressions Nouvelles, 2012 , p. 8.

${ }^{36}$ Ibidem.

${ }^{37}$ En outre, la méthode de recherche n'est pas non plus unifiée. C'est pourquoi Schoentjes parle des « écopoétiques » au pluriel car « une approche unifiée n'est ni envisageable ni même souhaitable» (SchoentJes Pierre, Ce qui a lieu. Essai d'écopoétique, op. cit., p. 276).

${ }^{38}$ FowlEs John, L'arbre, trad. François Rosso, Paris, Editions des Deux Terres, 2003 [1979], p. 36.

${ }^{39}$ Ibidem.

${ }^{40}$ Ibidem.
} 
Pour Barry Lopez, le secret de l'écriture de la nature réside dans le développement d'une attention intime, d'une relation sensorielle et d'une union éthique avec le monde naturel. La réciprocité qui s'instaure ainsi permettra à l'écrivain de s'ouvrir à son environnement. Il en résulte un sentiment d'interdépendance, car " a sense of place », l'expérience du lieu, " est essentielle au développement d'un sens de la moralité et de l'identité humaine ${ }^{41}$. L'auteur qui arrive à (faire) ressentir la nature de cette façon produira une littérature qui s'adresse à notre conscience environnementale puisque, comme l'affirme Gascar,

nos rapports avec la réalité quotidienne, la matière même, ne représentent-ils pas des intrigues constamment renouvelées? Nous débouchons ici sur l'aventure ontologique, poétique, si l'on préfère. Le thème entraîne le style, le détermine. Il y a, dans le roman élargi tel que je le conçois, une poésie préalable à l'écriture. En nommant le monde, on ne court guère le risque de mal écrire ${ }^{42}$.

\section{Bibliographie}

\section{Sources primaires}

CONRAD Joseph, Le nègre du « Narcisse », trad. Robert d'Humières, Paris, Gallimard, 1938 [1898].

FOWLES John, L'arbre, trad. François Rosso, Paris, Editions des Deux Terres, 2003 [1979].

GASCAR Pierre, Les bêtes suivi de Le temps des morts, Paris, Gallimard, 1953.

GASCAR Pierre, L'homme et l'animal, Paris, Albin Michel, 1974.

GASCAR Pierre, Les sources, Paris, Gallimard, 1975.

GASCAR Pierre, Le règne végétal, Paris, Gallimard, 1981.

GASCAR Pierre, Pour le dire avec des fleurs, Paris, Gallimard, 1988.

GASCAR Pierre, La friche, Paris, Gallimard, 1993.

GASCAR Pierre, «Une civilisation qui retourne à l'école des fleurs », in Pierre Gascar et Guy Rohou. Correspondance. 1963-1995, Bibliothèque d'Étude et de Conservation de Besançon, fonds Pierre Gascar, Ms. Z 481.

RoMAIN Gary, Éducation européenne, Paris, Éditions Gallimard, coll. « Folio », 1972 [1956].

\section{Sources secondaires}

ACQUIER Marie-Laure, «Présentation : soif de mots, désir de science. Quelques aspects de la relation entre littérature et science(s) », Cahiers de Narratologie, ${ }^{\circ} 18,2010$, mis en ligne le 16 juillet 2010, consulté le 20 octobre 2019. Disponible sur : http://narratologie.revues.org/6116.

BEKOFF Mark, Encyclopedia of animal rights and animal welfare. Second edition, Volume I : A-H, Santa Barbara (California), Greenwood Press, 2010.

BERTRAND Romain, Le détail du monde. L'art perdu de la description de la nature, Paris, Éditions du Seuil, 2019.

BLANC Nathalie, PUGHE Thomas, CHARTIER Denis, « Littérature \& Écologie : vers une écopoétique », Écologie et Politique, n³6, 2008, p. 15-28.

\footnotetext{
${ }^{41}$ LOPEZ Barry, «A Literature of Place », U.S. Society and Values, 10, n¹, août 1996, p. 10, nous traduisons.

${ }^{42}$ Pierre Gascar cité par RoHou Guy, «Pierre Gascar et les vérités de la nuit », La Nouvelle Revue française, n²21, mai 1971, p. 89.
} 
Chelebourg Christian, Les écofictions. Mythologies de la fin du monde, Bruxelles, Les Impressions Nouvelles, 2012.

BESS Michael, La France vert clair. Ecologie et modernité technologique 1960-2000, trad. Chr. Jaquet, Paris, Champ Vallon, 2011 [2003], p. 79.

BUEKENS Sara, « Pour que l'écologie supplante le nationalisme : l'esthétique de Pierre Gascar », Revue critique de fixxion française contemporaine, $\mathrm{n}^{\circ} 11$, «Écopoétiques », sous la dir. d'Alain Romestaing, Pierre Schoentjes et Anne Simon, décembre 2015, p. 49-59.

BUEKENS Sara, «L'écopoétique : une nouvelle approche de la littérature française », Elfe XX-XXI, n8, «Extension du domaine de la littérature », sous la dir. d'Alexandre Gefen et Claude Perez, 2019, mis en ligne le 15 septembre 2019, consulté le 20 octobre 2019. Disponible sur : http://journals.openedition.org/elfe/1299.

BUELL Lawrence, The Environmental Imagination, Cambridge/London, Harvard University Press, 1995.

CORVOL André, L'homme aux bois. Histoire des relations de l'homme et de la forêt XVII ${ }^{e}-X X^{e}$ siècle, Paris, Fayard, 1987.

CURATOla Bruno, «Les Bêtes de Pierre Gascar : la passion de l'être animal », in L'animal littéraire, Des animaux et des mots, sous la dir. de Jacques Poirier, Dijon, Éditions Universitaires de Dijon, 2010, p. 25-35.

HEISE Ursula, « Science and Ecocriticism », American Book Review, 18, n5, 1997, p. 4-6.

LeUTRAT Jean-Louis, Gracq, Paris, Editions Universitaires, coll. « Classiques du XXe siècle », 1967.

LOPEZ Barry, «A Literature of Place », U.S. Society and Values, 10, n¹, août 1996, p. 10-12.

PEYRE Henri, Six maîtres contemporains, New York, Harcourt, Brace \& World, 1969.

RobBE-GRILLET Alain, "Pierre Gascar : Le temps des morts (Gallimard) », La Nouvelle Revue française, ${ }^{\circ} 13$, janvier 1954, p. 137-138.

RoHOU Guy, «Pierre Gascar et les vérités de la nuit », La Nouvelle Revue française, ${ }^{\circ} 221$, mai 1971, p. 88-93.

SCHOENTJES Pierre, «Pierre Gascar: retour sur Le Temps des morts », in Mémoires occupées. Fictions françaises et Seconde Guerre mondiale, sous la dir. de Marc Dambre, Paris, Presses Sorbonne Nouvelle, 2013, p. 101-110.

SChoentJes Pierre, Ce qui a lieu. Essai d'écopoétique, Marseille, Éditions Wildproject, 2015.

TERMITE Marinella, Le sentiment végétal, Macerata, Quodlibet, 2014. 